\title{
The effect of radiation on MHD natural convection flow of fluid with variable viscosity from a porous vertical plate
}

\author{
Amena Ferdousi ${ }^{1 *}$, M. Mostafizur Rahman ${ }^{2}$, Mohammad Salek Parvez $^{3}$, M. A. Alim ${ }^{4}$ \\ ${ }^{1}$ Faculty of Electronics and Electrical Engineering, Eastern University, Dhaka, Bangladesh \\ ${ }^{2}$ Department of Computer Science \& Engineering, Daffodil International University, Dhaka, Bangladesh \\ ${ }^{3}$ Department of Computer Science \& Engineering, Daffodil International University, Dhaka, Bangladesh \\ ${ }^{4}$ Department of Mathematics, Bangladesh University of Engineering and Technology, Dhaka, Bangladesh \\ *Corresponding author E-mail: amena@easternuni.edu.bd
}

\begin{abstract}
This paper presents a new extension for the effect of Radiation on MHD Natural Convection Flow with Variable Viscosity from a Porous Vertical Plate. The governing boundary layer equations are first transformed into a non dimensional form and the resulting non linear system of partial differential equations are then solved numerically using finite difference method together with Keller-Box scheme. The numerical results show that the variable viscosity affects the surface shear stress and the rate of heat transfer which are here in terms of skin friction coefficient and local Nusselt number. It affects velocity as well as temperature profiles also. These are shown graphically and tabular form for a selection of parameters set of consisting of viscosity variation parameter $\gamma$, magnetohydrodynamic parameter $M$, radiation effect $R_{d}$, Prandtl number $P r$.
\end{abstract}

Keywords: Radiation effect, Porous plate, Magnetohydrodynamics, Natural convection, variable viscosity

\section{Introduction}

The study of the flow of electrically conducting fluid in presence of magnetic field is also important from the technical point of view and such types of problems have received much attention by many researchers. The specific problem selected for study is the flow and heat transfer in an electrically conducting fluid adjacent to the surface. The interaction of the magnetic field and the moving electric charge carried by the flowing fluid induces a force, which tends to oppose the fluid motion. And near the leading edge the velocity is very small so that the magnetic force, which is proportional to the magnitude of the longitudinal velocity and acts in the opposite direction is also very small. Consequently the influence of the magnetic field on the boundary layer is exerted only through induced forces within the boundary layer itself, with no additional effects arising from the free stream pressure gradient . MHD was originally applied to astrophysical and geophysical problems but more recently to the problem of fusion power, where the application is the creation and containment of hot plasmas by electromagnetic forces, since material wall be destroyed. Astrophysical problems include solar structure especially in the outer layers, the solar wind bathing the earth and other planets and interstellar magnetic fields.

The effect of radiation on MHD free convection flow with variable viscosity from porous vertical plate has been drawn forth not only for its fundamental aspects but also for its significance in the contexts of space technology and processes involving high temperature. In the presence variable viscosity on MHD free convection boundary layer flow from a porous vertical plate of a steady two dimensional viscous incompressible fluid and the radiated heat transfer has been investigated. In this analysis consideration had been given to grey gases that emit and absorb but do not scatter thermal radiation. Over the work it is assumed that the surface temperature of the porous vertical plate, $T_{w}$, is constant, where $T_{w}>T_{\infty}$. Here $T_{\infty}$ is the ambient temperature of the fluid, $T$ is the temperature of the fluid in the boundary layer, $g$ is the acceleration due to gravity, the fluid is assumed to be a grey emitting and absorbing, but non scattering medium. In the present work following assumptions are made:

- The radiative heat flux in the $x$-direction is considered negligible in comparison with that in the $y$ direction, where the physical coordinates $(u, v)$ are velocity components along the $(x, y)$ axes.

- The physical property, variable viscosity may change significantly with temperature. 
Merkin [1] concluded free convection with blowing and suction. Lin and Yu [2] studied free convection on a horizontal plate with blowing and suction. Hossain et al [3] studied the effect of radiation on free convection flow with variable viscosity from a porous vertical plate. Hossain et al [4] performed flow of viscous incompressible fluid with temperature dependent viscosity and thermal conductivity past a permeable wedge with variable heat flux. Hossain and Takhar [5] studied radiation effect on mixed convection along a vertical plate with uniform surface temperature. Molla et al.. [6] studied natural convection flow along a vertical wavy surface with uniform surface temperature in presence of heat generation/absorption. Akhter [7] studied the effect of radiations on free convection flow on sphere with isothermal surface and uniform heat flux. Ali [8] studied the effect of radiation on free convection flow on sphere with heat generation. Hossain et al. [9] studied the effect of radiation on free convection flow from a porous vertical plate. They [9] analyzed a full numerical solution and found an increase in Radiation parameter $R_{d}$ causes to thin the boundary layer and an increase in surface temperature parameter causes to thicken the boundary layer. The presence of suction ensures that its ultimate fate if vertically increased is a layer of constant thickness. Vajravelu and Hadjinicolaou [10] perfomed the heat transfer in a viscous fluid over a stretching sheet with viscous dissipation and internal heat generation In this study, they considered that the volumetric rate of heat generation, $q^{m}\left[\mathrm{~W} / \mathrm{m}^{3}\right]$ should be

$$
q^{m}=\left\{\begin{array}{cc}
Q_{0}\left(T-T_{\infty}\right) & \text { for } T \geq T_{\infty} \\
0 & \text { for } T<T_{\infty}
\end{array}\right.
$$

where $Q_{0}$ is the heat generation constant. The above relation explained is valid as an approximation of the state of some exothermic process and having $T_{\infty}$ as the onset temperature. When the inlet temperature is not less than $T_{\infty}$ they use $Q_{0}\left(T-T_{\infty}\right)$. Molla et al. [11] studied the Magnetohydrodynamic natural convection flow on a sphere with uniform heat flux in presence of heat generation. Accordingly, Gary et al. [12] and Mehta and Sood [13] have concluded that when this effect is included, the flow characteristics substantially change compared to the constant viscosity case. Recently, Kafoussius and Williams [14] and Kafoussias and Rees [15] have investigated the effect of the temperature-dependent viscosity on the mixed convection flow past a vertical flat plate in the region near the leading edge using the local nonsimilarity method. In these studies, they concluded that when the viscosity of a fluid is sensitive to temperature variations, the effect of temperature-dependent viscosity has to be taken into consideration; otherwise considerable errors may occur in the characteristics of the heat transfer process. Hossain and Kabir [16] have investigated the natural convection flow from a vertical wavy surface. Hossain and Munir [17] investigated the mixed convection flow from a vertical flat plate for a temperature dependent viscosity. In the studies $[16,17]$ the viscosity of the fluid has been considered to be inversely proportional to a linear function of temperature. In all the above studies were confined without any effect of radiation and heat generation.

None of the aforementioned studies, considered variable viscosity and MHD effects on laminar boundary layer flow of the fluids along porous plate with radiation heat loss.

In the present study, we have investigated the effects of radiation with variable viscosity on MHD natural convection flow from a porous vertical plate numerically. The results will be obtained for different values of relevant physical parameters and will be shown in graphs as well as in tables.

The governing partial differential equations are reduced to locally non-similar partial differential forms by adopting some appropriate transformations. The transformed boundary layer equations are solved numerically using implicit finite difference scheme together with the Keller box technique [20]. Here, we have focused our attention on the evolution of the surface shear stress in terms of local skin friction and the rate of heat transfer in terms of local Nusselt number, velocity profiles as well as temperature profiles for selected values of parameters consisting of MHD $M$, variable viscosity $\gamma$, Prandtl number $\operatorname{Pr}$ and the radiation parameter $R_{d}$.

\section{Problem description and mathematical model}

We have investigated the effect of radiation with variable viscosity on MHD free convection flow from a porous plate. The fluid is assumed to be a grey, emitting and absorbing but non scattering medium. Over the work it is assumed that the surface temperature of the porous vertical plate, $T_{w}$, is constant, where $T_{w}>T_{\infty}$. The physical configuration considered is as shown in Fig.1:

The conservation equations for the flow characterized with steady, laminar and two dimensional boundary layer; under the usual Boussinesq approximation, the continuity, momentum and energy equations can be written as:

$$
\frac{\partial u}{\partial x}+\frac{\partial v}{\partial y}=0
$$




$$
\begin{aligned}
& \rho\left(u \frac{\partial u}{\partial x}+v \frac{\partial u}{\partial y}\right)=\frac{\partial}{\partial y}\left(\mu \frac{\partial u}{\partial y}\right)+\rho g \beta\left(T-T_{\infty}\right) \\
& \rho c_{p}\left(u \frac{\partial T}{\partial x}+v \frac{\partial T}{\partial y}\right)=k \frac{\partial^{2} T}{\partial y^{2}}-\frac{\partial q_{r}}{\partial y}+Q_{0}\left(T-T_{\infty}\right)
\end{aligned}
$$

With the boundary conditions

$$
\begin{aligned}
& x=0, y>0, u=0, T=T_{\infty} . \\
& y=0, x>0, u=0, v=-V, T=T_{w} \\
& y \rightarrow \infty, x>0, u=0, T=T_{\infty}
\end{aligned}
$$

where $\rho$ is the density, $\beta_{0}$ is the strength of magnetic field, $\sigma_{0}$ is the electrical conduction, $k$ is the thermal conductivity, $\beta$ is the coefficient of thermal expansion, $v$ is the reference kinematic viscosity $v=\mu \rho, \mu$ is the viscosity of the fluid, $C_{p}$ is the specific heat due to constant pressure and $q_{r}$ is the radiative heat flux in the $y$ direction. In order to reduce the complexity of the problem and to provide a means of comparison with future studies that will employ a more detail representation for the radiative heat flux; we will consider the optically thick radiation limit. Thus radiation heat flux term is simplified by the Rosseland diffusion approximation Ozisik (1973) [19] and is given by

$$
q_{r}=-\frac{4 \sigma}{3\left(a_{r}+\sigma_{s}\right)} \frac{\partial T^{4}}{\partial y}
$$

In Equation (5) $a_{r}$ is the Rosseland mean absorption co-efficient, $\sigma_{\mathrm{s}}$ is the scattering co-efficient and $\sigma$ is the StephanBoltzman constant.

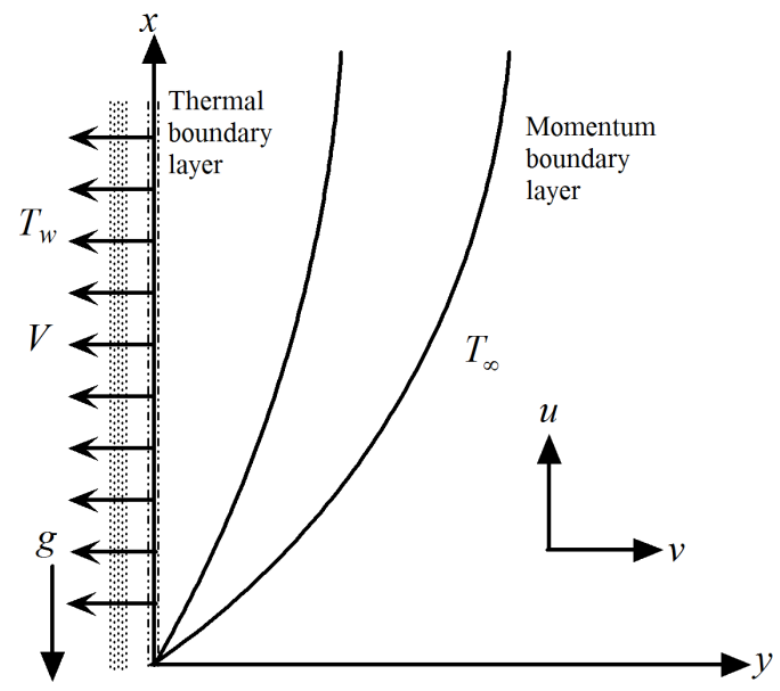

Fig. 1: The coordinate system and the physical model

In Equation (5a) $a_{r}$ is the Rosseland mean absorption co-efficient, $\sigma_{\mathrm{s}}$ is the scattering co-efficient and $\sigma$ is the StephanBoltzman constant.

The absolute viscosity $\mu$ is assumed to be vary with temperature according to a general functional form $\mu=\mu_{\mathrm{f}} \mathrm{s}(\mathrm{T})$, where $\mu_{\mathrm{f}}$ is the absolute viscosity at the film temperature $\mathrm{T}_{\mathrm{f}}$ and $\mathrm{s}\left(\mathrm{T}_{\mathrm{f}}\right)=1$. This form is chosen to allow definition of the stream function based on the absolute viscosity at the film temperature. For liquids, all transport properties vary with temperature. However, for many liquids, petroleum oils, glycerin, glycol, silicon fluids and some molten salt, the percent variation of absolute viscosity with temperature is much more than that of the other properties. Under the above conditions an analysis incorporating the above assumptions and describing the momentum and thermal transport within the flow field are more accurate than the usual assumption of constant properties evaluated at some reference temperature. It should be mentioned here that there are some fluids for which properties other than $\mu$ vary strongly with temperature. In particular, water and methyl alcohol exhibit strong variation of both $\mu$ and $\beta$. The analysis presented here is not applicable to these liquids since we are considering only the variation of the absolute viscosity as a function of temperature. However, for the case of an isothermal surface (in an unstratified ambient fluid), the variation of the absolute viscosity with temperature takes the form $\mu=\mu_{\mathrm{f}} S(\theta)$, where $\theta$ is the dimensionless temperature in the boundary layer defined in equation (5b), such that $S(1 / 2)=1$. A wide variety of functional forms of $S(\theta)$ satisfying this requirement was investigated in the literature such as algebraic expressions, power series, exponential forms, etc. 
Following Carey and Mollendorf [18], the simplest form of the absolute viscosity is used in this investigation as follows:

$\mu=\mu_{f}\left[1+\frac{1}{\mu_{f}}\left(\frac{d \mu}{d t}\right)_{f}\left(T-T_{\infty}\right)\right]$

This simple form amounts to a linear variation of the absolute viscosity with temperature, with the slope $\mathrm{d} \mu / \mathrm{d} T$, evaluated at film temperature. The assumed linear variation of viscosity with temperature gives rise to a new parameter $\gamma$ defined by

$\gamma=\frac{1}{\mu_{f}}\left(\frac{d \mu}{d t}\right)_{f}\left(T-T_{\infty}\right)$

Now introduce the following non-dimensional variables:

$$
\begin{aligned}
& \eta=\frac{V y}{v \xi}, \xi=V\left\{\frac{4 x}{v^{2} g \beta \Delta T}\right\}^{\frac{1}{4}}, \psi=V^{-3} v^{2} g \beta \Delta T \xi^{3}\left\{f+\frac{\xi}{4}\right\} \\
& \theta=\frac{T-T_{\infty}}{T_{w}-T_{\infty}}, \theta_{w}=\frac{T_{w}}{T_{\infty}}, \quad \Delta=\theta_{w}-1=\frac{T_{w}}{T_{\infty}}-1=\frac{T_{w}-T_{\infty}}{T_{\infty}}, R d=\frac{4 \sigma T_{\infty}^{3}}{k\left(a+\sigma_{S}\right)}
\end{aligned}
$$

Where, $\theta$ is the non-dimensional temperature function, $\theta_{w}$ is the surface temperature parameter and $R_{d}$ is the radiation parameter.

Substituting (6) into Equations (1), (2) and (3) leads to the following non-dimensional equations

$$
\begin{aligned}
& {\left[1+\gamma\left(\theta-\frac{1}{2}\right)\right] f^{\prime \prime \prime}+\theta-2 f^{\prime 2}+3 f f^{\prime \prime}+\xi f^{\prime \prime}=\xi\left(f^{\prime} \frac{\partial f^{\prime}}{\partial \xi} f^{\prime \prime} \frac{\partial f^{\prime}}{\partial \xi}\right)} \\
& \frac{1}{\operatorname{Pr}} \frac{\partial}{\partial \eta}\left[\left\{1+\frac{4}{3} R d\left(1+\left(\theta_{w}-1\right) \theta\right)^{3}\right\} \frac{\partial \theta}{\partial \eta}\right]+Q \theta \xi^{2}+3 f \theta^{\prime}+\xi \theta^{\prime}=\xi\left(f f^{\prime} \frac{\partial \theta}{\partial \xi}-\frac{\partial f}{\partial \xi} \theta^{\prime}\right)
\end{aligned}
$$

Where $\operatorname{Pr}=v C_{p} / k$ is the Prandtl number and $Q=v Q_{0} / v^{2} \rho C_{p}$ is the heat generation parameter .

The boundary conditions (4) become

$f=0, f^{\prime}=0, \theta=1$ at $\eta=0$

$f^{\prime}=0, \theta=0$ as $\eta \rightarrow \infty$

The solution of equations (6), (8) enable us to calculate the nondimensional velocity components $\bar{u}, \bar{v}$ from the following expressions

$$
\begin{aligned}
& \bar{u}=\frac{v^{2}}{V g \beta\left(T_{w}-T_{\infty}\right)} u=\xi^{2} f^{\prime}(\xi, \eta) \\
& \bar{v}=\frac{v}{V}=\xi^{-1}\left(3 f+\xi-\eta f^{\prime}+\xi \frac{\partial f}{\partial \xi}\right)
\end{aligned}
$$

In practical applications, the physical quantities of principle interest are the shearing stress $\tau_{\mathrm{w}}$ and the rate of heat transfer in terms of the skin-friction coefficients $C_{f x}$ and Nusselt number $N u_{x}$ respectively, which can be written as

$$
\begin{aligned}
& N u_{x}=\frac{v}{V \Delta T}\left(q_{c}+q_{r}{ }^{\prime}{ }_{\eta=0}, C_{f x}=\frac{V}{g \beta \Delta T}(\tau)_{\eta=0}\right. \\
& \text { where } \tau_{w}=\mu\left(\frac{\partial u}{\partial y}\right)_{\eta=0} \text { and } q_{c}=-k\left(\frac{\partial T}{\partial y}\right)_{\eta=0}
\end{aligned}
$$

$q_{c}$ is the conduction heat flux.

Using the Equations (6) and the boundary condition (9) into (11) and (12), we get

$$
\begin{aligned}
C_{f x} & =\xi\left(1+\frac{\gamma}{2}\right) f^{\prime \prime}(x, 0) \\
N u_{x} & =\xi^{-1}\left(1+\frac{4}{3} R_{d} \theta_{w}^{3}\right) \theta^{\prime}(x, 0)
\end{aligned}
$$

The values of the velocity and temperature distribution are calculated respectively from the following relations: $\bar{u}=\xi^{2} f^{\prime}(\xi, \eta), \quad \theta=\theta(x, y)$ 


\section{Numerical procedure}

Solution of the local non similar partial differential equation (7) to (8) subjected to the boundary condition (9) are obtained by using implicit finite difference method with Keller-Box Scheme [20], which has been described in details by Cebeci [21].

The solution methodology of equations (7) and (8) with the boundary condition given in eqn. (9) for the entire $\xi$ values based on Keller - box scheme is proposed here. The scheme specifically incorporated a nodal distribution favoring the vicinity of the plate, enabling accuracy to be maintained in this region of steep gradient. In detail equations (7) and (8) are solved as a set of five simultaneous equations.

$\left[1+\gamma\left(\theta-\frac{1}{2}\right) f^{\prime \prime}\right]^{\prime}+3 f f^{\prime \prime}-2\left(f^{\prime}\right)^{2}+\theta-\xi f^{\prime \prime}=\xi\left(f^{\prime} \frac{\partial f^{\prime}}{\partial \xi}-\frac{\partial f}{\partial \xi} f^{\prime \prime}\right)$

and

$$
\frac{1}{\operatorname{Pr}} \frac{\partial}{\partial \eta}\left[\left\{1+\frac{4}{3} R d\left(1+\left(\theta_{w}-1\right) \theta\right)^{3}\right\} \frac{\partial \theta}{\partial \eta}\right]+Q \theta \xi^{2}+3 f \theta^{\prime}+\xi \theta^{\prime}=\xi\left(f^{\prime} \frac{\partial \theta}{\partial \xi}-\frac{\partial f}{\partial \xi} \theta^{\prime}\right)
$$

To apply the aforementioned method, we first convert Equations (15)-(16) into the following system of first order equations with dependent variables $u(\xi, \eta), v(\xi, \eta), p(\xi, \eta)$ and $g(\xi, \eta)$ as

$f^{\prime \prime}=u, u^{\prime}=v, \quad g=\theta$, and $\theta^{\prime}=p$

$$
\begin{aligned}
& {\left[1+p_{5}\left(g-\frac{1}{2}\right) v\right]^{\prime}+p_{1} f v-p_{2} u^{2}+g-\xi v=\xi\left(u \frac{\partial u}{\partial \xi}-\frac{\partial f}{\partial \xi} v\right)} \\
& \frac{1}{\operatorname{Pr}} \frac{\partial}{\partial \eta}\left[\left\{1+p_{3}(1+\Delta g)^{3}\right\} p\right]+p_{4} g \xi^{2}+\xi p+p_{1} f p=\xi\left(u \frac{\partial g}{\partial \xi}-p \frac{\partial f}{\partial \xi}\right) \\
& \frac{1}{\operatorname{Pr}}\left[p^{\prime}+\left(p_{3} p(1+\Delta g)\right\}^{3}\right]+p_{4} g \xi^{2}+\xi p+p_{1} f p=\xi\left(u \frac{\partial g}{\partial \xi}-p \frac{\partial f}{\partial \xi}\right)
\end{aligned}
$$

where

$p_{1}=3, p_{2}=2, p_{3}=\frac{4}{3} R d, p_{4}=\mathrm{Q}$ and $p_{5}=\gamma$

The corresponding boundary conditions are

$f(\xi, 0)=0, u(\xi, 0)=0$ and $g(\xi, 0)=0$

$u(\xi, \infty)=0, g(\xi, \infty)=0$

We now consider the net rectangle on the $(\xi, \eta)$ plane and denote the net point by

$\eta_{0}=0, \quad \eta_{j}=\eta_{j-1}+h_{j}, \quad j=1,2, \ldots J$

$\xi^{0}=0, \quad \xi^{n}=\xi^{n-1}+k_{n}, \quad n=1,2, \ldots \quad N$

We approximate the quantities $(f, u, v, p)$ at the points $\left(\xi^{n}, \eta_{j}\right)$ of the net by $\left(f_{j}^{n}, u_{j}^{n}, v{ }_{j}^{n}, p_{j}^{n}\right)$ which we call net function.

$\eta_{j-1 / 2}=\frac{1}{2}\left(\eta_{j}-\eta_{j-1}\right)$

$\xi^{n-1 / 2}=\frac{1}{2}\left(\xi^{n}+\xi^{n-1}\right)$

$g_{j}{ }^{n-1 / 2}=\frac{1}{2}\left(g_{j}^{n}+g_{j}^{n-1}\right)$

$g_{j-1 / 2}^{n}=\frac{1}{2}\left(g_{j}^{n}+g_{j-1}^{n}\right)$

Now we write the difference equations that are to approximate Equations (17) - (19) by considering one mesh rectangle for the mid point $\left(\xi^{n},{ }_{j-1 / 2}\right)$ to obtain

$\frac{f_{j}^{n}-f_{j-1}^{n}}{h_{j}}=u_{j-1 / 2}^{n}$

$\frac{u_{j}^{n}-u_{j-1}^{n}}{h_{j}}=v_{j-1 / 2}^{n}$ 
$\frac{g_{j}^{n}-g_{j-1}^{n}}{h_{j}}=p_{j-1 / 2}^{n}$

Similarly Equations (18) - (19) are approximate by centering about the midpoint $\left(\xi^{n-1 / 2},{ }_{j-1 / 2}\right)$. Centering the Equations (22) about the point $\left(\xi^{n-1 / 2}, n\right)$ without specifying $\eta$ to obtain the algebraic equations. The difference approximation to Equations (18)-(19) become

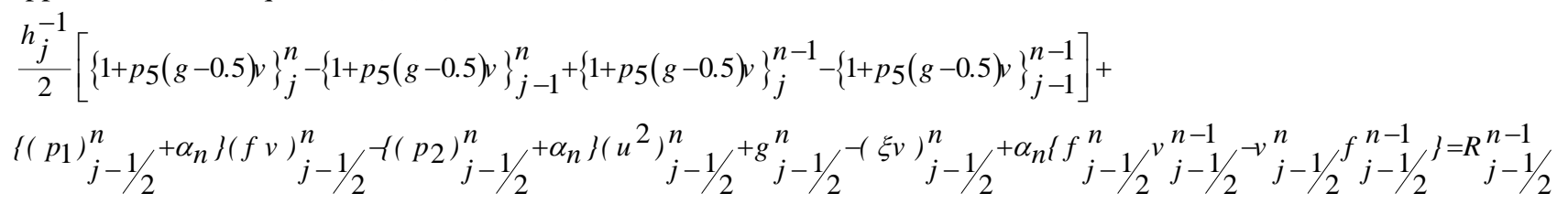

where

$\underset{j-\frac{1}{2}}{L^{n-1}}=h_{j}^{-1}\left[\left\{1+p_{5}(g-0.5)\right\}_{j}^{n-1}-\left\{1+p_{5}(g-0.5)\right\}_{j-1}^{n}\right]+\left(p_{1}\right)_{j-\frac{1}{2}}^{n-1}(f v)_{j-\frac{1}{2}}^{n-1}-\left(p_{2}\right)_{j-\frac{1}{2}}^{n-1}\left(u^{2}\right)_{j-\frac{1}{2}}^{n-1}{ }_{j-\frac{1}{2}}^{n-1}-(\xi p)_{j-\frac{1}{2}}^{n-1}+h_{j}^{-1}\left(v_{j}^{n-1}-v_{j-1}^{n-1}\right)$

And $\quad R_{j-\frac{1}{2}}^{n-1}=-L_{j-\frac{1}{2}}^{n-1}+\alpha_{n}\left\{-\left(u^{2}\right)^{n-1}+\frac{1}{2}+(f v)_{j-\frac{1}{2}}^{n-1}\right\}$

$\frac{1}{\operatorname{Pr}}\left[h_{j}^{-1}\left(p_{j}^{n}-p_{j-1}^{n}\right)+h_{j}^{-1}\left\{\left\{p_{3} p(1+\Delta g)^{3}\right\}_{j}^{n}-\left\{p_{3} p(1+4 g)^{3}\right\}_{j-1}^{n}\right\}\right]+$

$\xi_{j-1 / 2}^{n} p_{j-1 / 2}^{n}+\left(p_{4}\right)_{j-1 / 2}^{n} g_{j-1 / 2}^{n}+\left(p_{1}\right)_{j-1 / 2}^{n}(f p)_{j-1 / 2}^{n}$

$=\alpha n\left[\left\{(u g)_{j-1 / 2}^{n}-(u g)_{j-1 / 2}^{n-1}-u_{j-1 / 2}^{n} g_{j-1 / 2}^{n-1}+u_{j-1 / 2}^{n-1} g_{j-1 / 2}^{n}\right\}-\right.$

$\left.\left\{(f p)_{j-1 / 2}^{n}-(f p)_{j-1 / 2}^{n-1}-p_{j-1 / 2}^{n} f_{j-1 / 2}^{n-1}+p_{j-1 / 2}^{n-1} f_{j-1 / 2}^{n}\right\}\right]$

$-\frac{1}{\operatorname{Pr}}\left[h_{j}^{-1}\left(p_{j}^{n-1}-p_{j-1}^{n-1}\right)+h_{j}^{-1}\left\{\left\{p_{3} p(1+4 g)^{3}\right\}_{j}^{n-1}-\left\{p_{3} p(1+4 g)^{3}\right\}_{j}^{n-1}\right\}\right]-$

$\left[\xi_{j-1 / 2}^{n-1} p_{j-1 / 2}^{n-1}+\left(p_{4}\right)_{j-1 / 2}^{n-1} g_{j-1 / 2}^{n-1}+\left(p_{1}\right)_{j-1 / 2}^{n-1}(f p)_{j-1 / 2}^{n-1}\right]^{n}$

$\Rightarrow \frac{1}{P r}\left[h_{j}^{-1}\left(p_{j}^{n}-p_{j-1}^{n}\right)+h_{j}^{-1}\left\{\left\{p_{3} p(1+\Delta g)^{3}\right\}_{j}^{n}-\left\{p_{3} p(1+\Delta g)^{3}\right\}_{j-1}^{n}\right\}\right]+\xi_{j-1 / 2}^{n}{ }_{j-1 / 2}^{n}{ }^{+}$

$\left(p_{4} \xi^{2}\right)_{j-1 / 2}^{n}{ }_{j-1 / 2}^{n}+\left(p_{1}\right)_{j-1 / 2}^{n}(f p)_{j-1 / 2}^{n}=-M{ }_{j-1 / 2}^{n-1}+\alpha n\left[-(u g)_{j-1 / 2}^{n-1}+(f p)_{j-1 / 2}^{n-1}\right]+$

$\alpha_{n}\left[(u g)_{j-1 / 2}^{n}-(f p)_{j-1 / 2}^{n}{ }_{j-1 / 2}^{n}{ }_{j-1 / 2}^{n-1}+u_{j-1 / 2}^{n-1}{ }_{j-1 / 2}^{n}{ }_{j-1 / 2}^{j}+p_{j-1 / 2}^{n} p_{j-1 / 2}^{n-1}{ }_{j-1 / 2}^{n-1}\right]^{n}$

$\Rightarrow \frac{1}{\operatorname{Pr}}\left[h_{j}^{-1}\left(p_{j}^{n}-p_{j-1}^{n}\right)+h_{j}^{-1}\left\{\left\{p_{3} p(1+\Delta g)^{3}\right\}_{j}^{n}-\left\{p_{3} p(1+\Delta g)^{3}\right\}_{j-1}^{n}\right\}\right]+\xi_{j-1 / 2}^{n} p_{j-1 / 2}^{n}+$

$\left.\left(p_{4} \xi^{2}\right)_{j-1 / 2}^{n}{ }_{j-1 / 2}^{n}+\left(p_{1}\right)_{j-1 / 2}^{n}+\alpha_{n}\right)(f p)_{j-1 / 2}^{n}$

$-\alpha_{n}\left[\left\{(u g)_{j-1 / 2}^{n}-(u g)_{j-1 / 2}^{n-1}{ }_{j-1 / 2}^{n}{ }_{j-1 / 2}^{n-1}{ }_{j-1 / 2}^{+1}{ }_{j-1 / 2}^{n-1}{ }_{j-1 / 2}^{n+p_{j-1 / 2}^{n}}{ }_{j-1 / 2}^{n-1}{ }_{j-1 / 2}^{n-1} f_{j}^{n}\right.\right.$

$=T \begin{aligned} & n-1 \\ & j-1 / 2\end{aligned}$ 
where

$$
\begin{aligned}
& M_{j-1 / 2}^{n-1}=\frac{1}{\operatorname{Pr}}\left[h_{j}^{-1}\left(p_{j}^{n-1}-p_{j-1}^{n-1}\right)+h_{j}^{-1}\left\{\left\{p_{3} p(1+\Delta g)^{3}\right\}_{j}^{n-1}-\left\{p_{3} p(1+\Delta g)^{3}\right\}_{j}^{n-1}\right\}\right]- \\
& \left.{ }_{j-1 / 2}^{\xi^{n-1}}{ }_{j-1 / 2}^{n-1}+\left(p_{4} \xi_{2}\right)_{j-1 / 2}^{n-1} \stackrel{j-1 / 2}{n-1}_{j-\left(p_{1}\right)_{j-1 / 2}^{n-1}}^{(f p)_{j-1 / 2}^{n-1}}\right] \\
& T_{j-1 / 2}^{n-1}=-M_{j-1 / 2}^{n-1}+\alpha_{n}\left[(f p){ }_{j-1 / 2}^{n-1}-(\text { ug })_{j-1 / 2}^{n-1}\right]
\end{aligned}
$$

The corresponding boundary conditions (21) become

$f_{0}^{n}=0, \quad u_{0}^{n}=0, \quad g_{0}^{n}=1$

which just express the requirement for the boundary conditions to remain during the iteration process. Now we will convert the momentum and energy equations into system of linear Equations and together with the boundary conditions can be written in matrix or vector form, where the coefficient matrix has a block tri-diagonal structure. The whole procedure, namely reduction to first order followed by central difference approximations, Newton's quasi-linearization method and the block Thomas algorithm, is well known as the Keller- box method. $u_{J}^{n}=0, g_{J}^{n}=0$

\section{Results}

In this exertion the effect of radiation with variable viscosity on MHD natural convection flow from a porous vertical plate is investigated. Numerical values of local rate of heat transfer are calculated in terms of Nusselt number $N u_{x}$ for the surface of the porous vertical plate from lower stagnation point to upper stagnation point, for different values of the aforementioned parameters and these are shown in tabular form in Table 1 and Graphically in Figure 5-7. The effect for different values viscosity $\gamma$ on local skin friction coefficient $C_{f x}$ and the local Nusselt number $N u_{x}$, as well as velocity and temperature profiles are displayed in Fig. 2 to 7 . The aim of these figures are to display how the profiles vary in $\xi$, the selected streetwise co-ordinate.

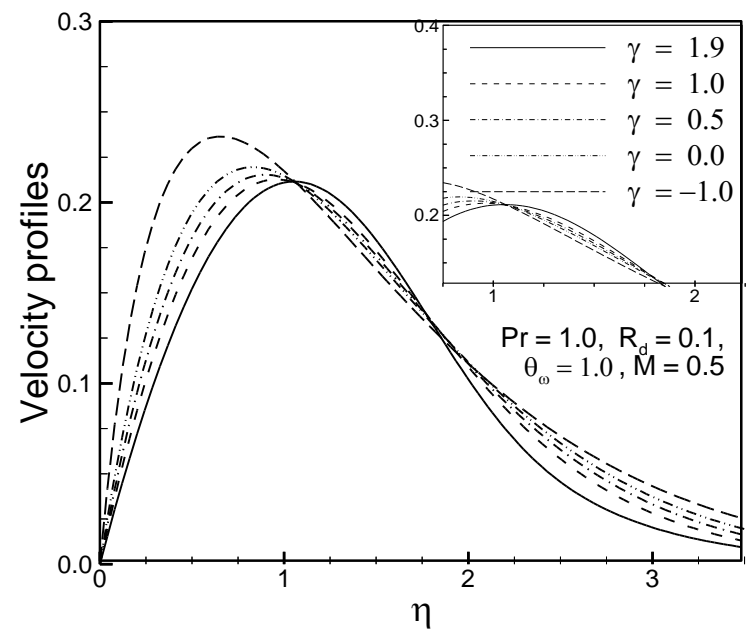

(a)

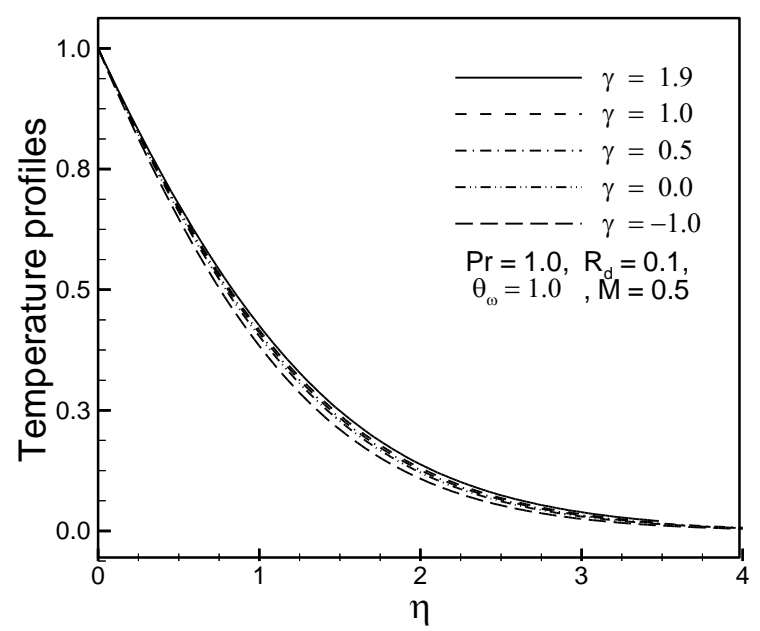

(b)

Fig 2. (a) Velocity and (b) temperature profiles for different values of viscosity parameter $\gamma$ with others fixed parameters.

Figures 2(a)-2(b) display results for the velocity and temperature profiles, for different values of viscosity parameter $\gamma$ $=-1.0,0.0,0.5,1.0,1.9$ while Prandtl number $\operatorname{Pr}=1.0$, radiation parameter $R_{d}=0.1$ surface temperature parameter $\theta_{\mathrm{w}}$ $=1.0$ and heat generation $M=0.5$. It has been seen from Figures 2(a)-2(b) that as the viscosity parameter $\gamma$ increases, the velocity profiles decreases and the temperature profiles decrease. The velocity is zero at the boundary wall then the velocity increases to the peak value as $\eta$ increases and from 1 to 1.8 it is reverse and after $\eta=1.8$ it is decreasing, finally the velocity approaches to zero (the asymptotic value).

The changes of temperature profiles in the $\eta$ direction also shows the typical temperature profiles for natural convection boundary layer flow that is the value of temperature profiles is 1.0 (one) at the boundary wall then the temperature profile decreases gradually along $\eta$ direction to the asymptotic value.

However, in figures 3(a)-3(b) it has been shown that when the Prandtl number $\operatorname{Pr}=0.8,2.0,4.0,6.0$ and 8.5 increases with $\theta_{\mathrm{w}}=1.0, R_{d}=0.1, M=0.5$ and $\gamma=1.0$ both the velocity and temperature profiles decrease. 


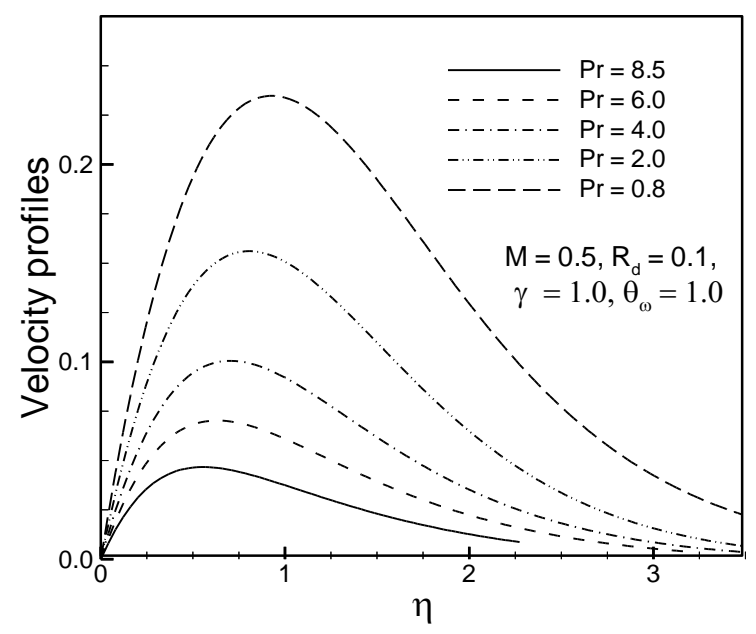

(a)

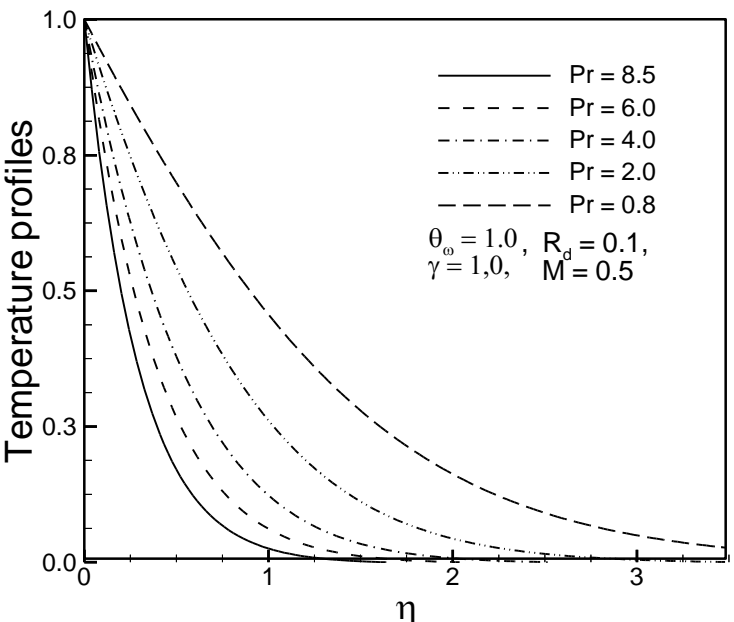

(b)

Fig 3. (a) Velocity and (b) temperature profiles for different values of prandtl number $P r$ with others fixed parameters.

Figures 4(a) display results for the velocity profiles for different values of MHD parameter $M$ with Prandtl number $P r=$ 1.0 , radiation parameter $R_{d}=0.1$, viscosity parameter $\gamma=1.0$ and surface temperature parameter $\theta_{\mathrm{w}}=1.0$. It has been seen from figure 4(a) that as the MHD parameter increases the velocity profiles decrease. It is also observed from figure 4(a) that the changes of velocity profiles in the $\eta$ direction reveals the typical velocity profile for natural convection boundary layer flow, i.e., the velocity is zero at the boundary wall then the velocity increases to the peak value as $\eta$ increases and finally the velocity approaches to zero (the asymptotic value). Figure 4(b) displays results for the temperature profiles, for different values of MHD parameter $M$ while Prandtl number $\operatorname{Pr}=1.0$, radiation parameter $R_{d}=0.1$, viscosity parameter $\gamma=1.0$ and surface temperature parameter $\theta_{\mathrm{w}}=1.0$. The maximum values of velocity are recorded to be 0.12853 at $\eta=0.83530$ and $0.15110,0.18095,0.19908,0.21952$ at $\eta=0.88811$ for $M=.30 .0$, 20.0,10.0, 5.0 and 0.0. The velocity is 0.21952 at $\eta=0.88811$ for $M=30.0$. Here, it is observed that at $\eta=0.88811$, the velocity decreases by $44.44 \%$ as the MHD parameter $M$ changes from 0 to 30.0 .

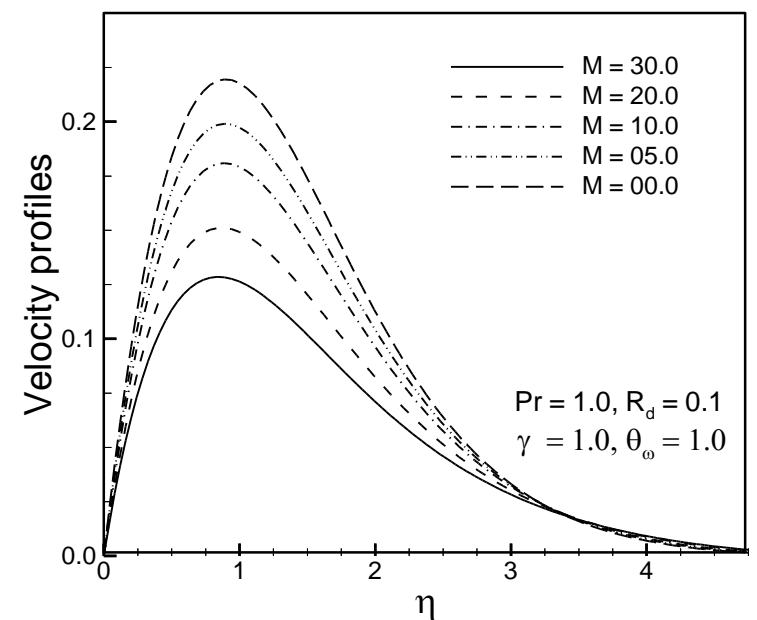

(a)

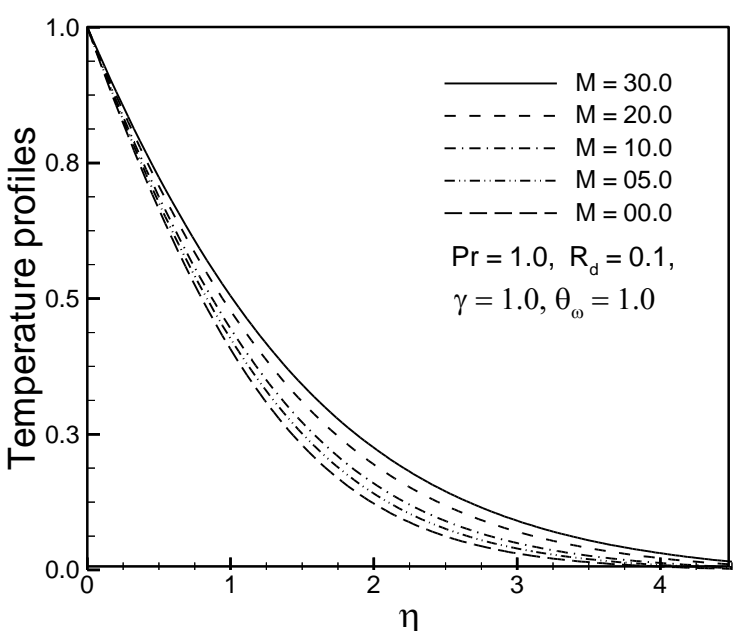

(b)

Fig. 4 (a) Velocity and (b) temperature profiles for different values of heat generation parameter $\mathrm{Q}$ with others fixed parameters.

From figure 4(b), as the MHD parameter $M$ increases, the temperature profiles increase. We observed that the temperature profile is 1.0 (one) at the boundary wall then the temperature profile decreases gradually along $\eta$ direction to the asymptotic value. But for $M=30.0,20.0,10.0,5.0,0.0$ the temperature profile increases, at $\eta=0.88811$ it is $0.548873,0.52355,0.49272,0.47532$ and 0.45685 then it decrease. And for other values it is gradually increasing.

Figure 5(a) shows that skin friction coefficient $C_{f x}$ increases for increasing values of viscosity parameter $\gamma$ with Prandtl number $\operatorname{Pr}=1.0$, radiation parameter $R_{d}=0.1$, surface temperature parameter $\theta_{\mathrm{w}}=1.0$ and $\mathrm{MHD}$ parameter $M=0.5 \mathrm{It}$ is observed from Figure 5(a) that the skin friction increases gradually from zero value at lower stagnation point along the $\xi$ direction and from Figure 5(b); it reveals that the rate of heat transfer decreases along the $\xi$ direction for $\gamma=-1.0,0.0$, $0.5,1.0$ and $1.9 N u_{x}$ are along $\xi$ axis. A hot fluid layer is created adjacent to the interface of the wall due to the 
viscosity mechanism and ultimately the resultant temperature of the fluid exceeds the surface temperature. Accordingly, the heat transfer rate from the surface decreases as shown in Fig. 5(b)

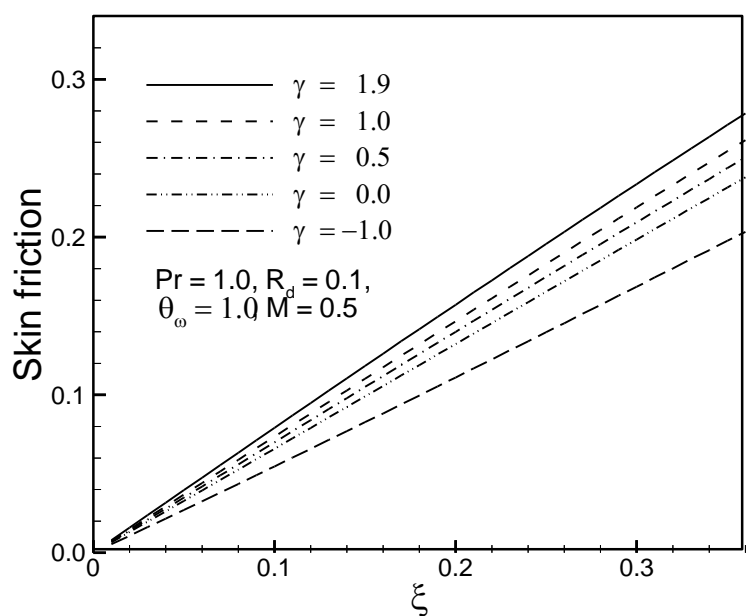

(a)

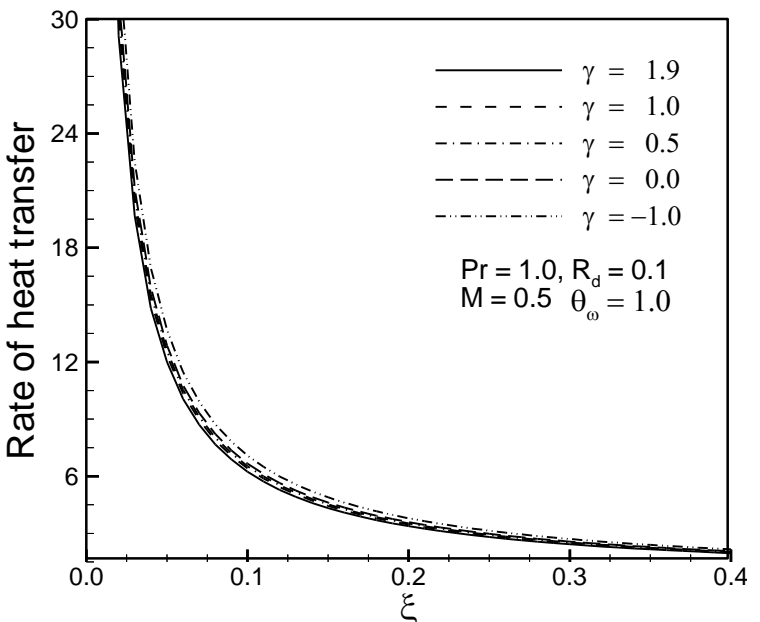

(b)

Fig 5.(a) Skin friction and (b) rate of heat transfer for different values of viscosity parameter $\gamma$ with others fixed parameters.

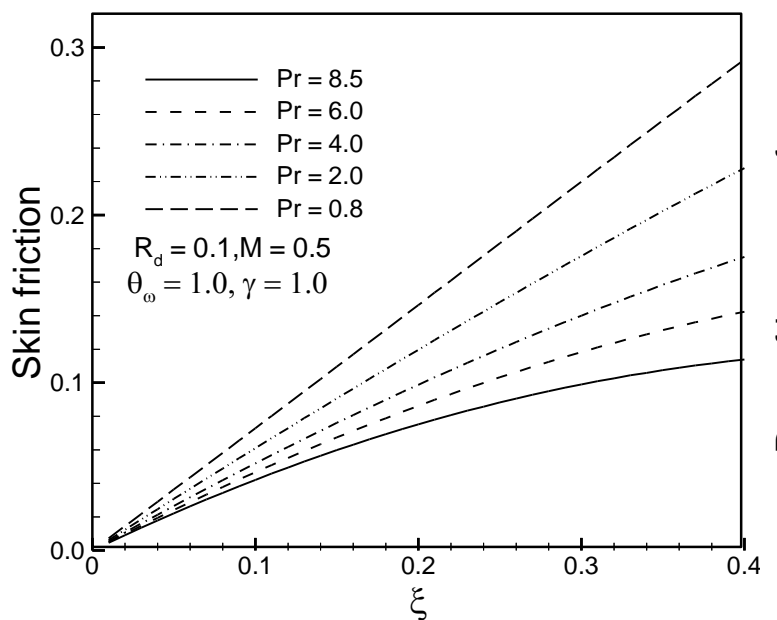

(a)

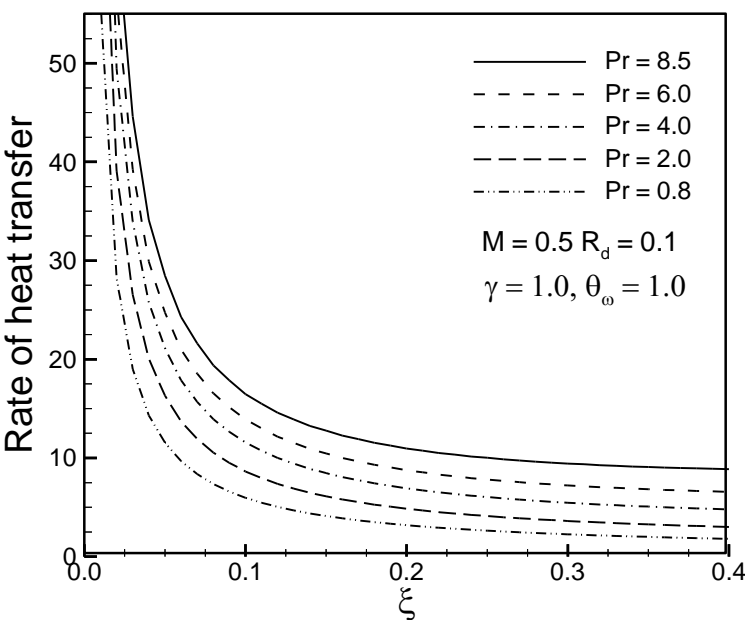

(b)

Fig 6.(a) Skin friction and (b) rate of heat transfer for different values of prandtl number $\operatorname{Pr}$ with others fixed parameters.

The variation of the local skin friction coefficient $C_{f x}$ and local rate of heat transfer $N u_{x}$ for different values of Prandtl number $\operatorname{Pr}$ while $\theta_{w}=1.0, R_{d}=0.1, M=0.5$ and $\gamma=1.0$ are shown in Figures 6(a)-6(b).

We can observe from these figures that as the Prandtl number $P r$ increases, the skin friction coefficient decreases and rate of heat transfer increases.

Figures 7(a)-7(b) show that skin friction coefficient $C_{f x}$ and heat transfer coefficient $N u_{x}$ decrease for increasing values of MHD parameter $M$ while viscosity parameter $\gamma=0.5$,. Prandtl number $P r=1.0$, radiation parameter $R_{d}=0.1$ and surface temperature parameter $\theta_{\mathrm{w}}=1.0$. The values of skin friction coefficient $C_{f x}$ and Nusselt number $N u_{x}$ are recorded to be $0.99889,0.51655,0.39677,0.29810 .0 .25075$ and $1.03770,1.00384,1.00388,1.00510$ and 0.99654 for $Q=$ $10.0,5.0,7.0 .2 .0,0.0$ and respectively which occur at the same point $\xi=1.7$. Here, it observed that at $\xi=1.7$, the skin friction decreases by $74.89 \%$ and Nusselt number $N u_{x}$ decreases by $4.13 \%$ as the MHD parameter $M$ changes from 0.0 to 30.0. It is observed from figure 7(a) that the skin friction increases gradually from zero value at lower stagnation point along the $\xi$ direction and from Figure 7(b); it reveals that the rate of heat transfer decreases along the $\xi$ direction. 


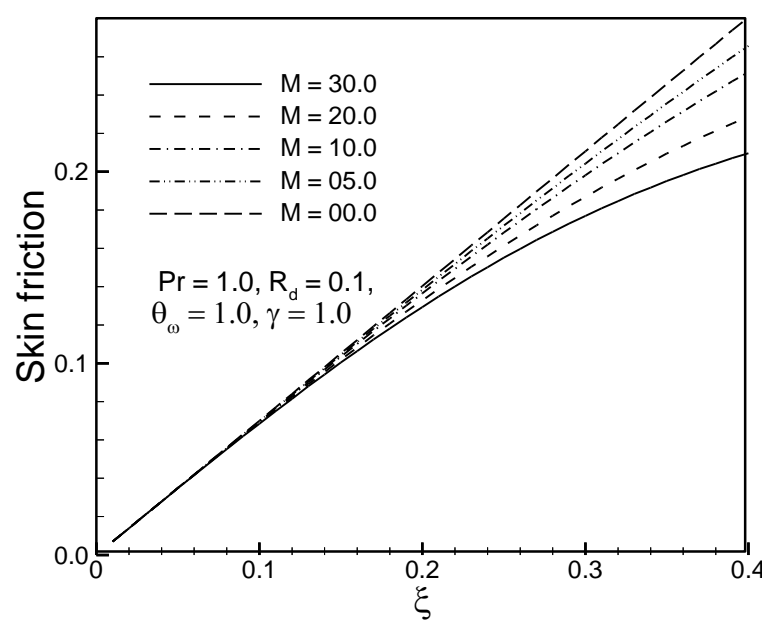

(a)

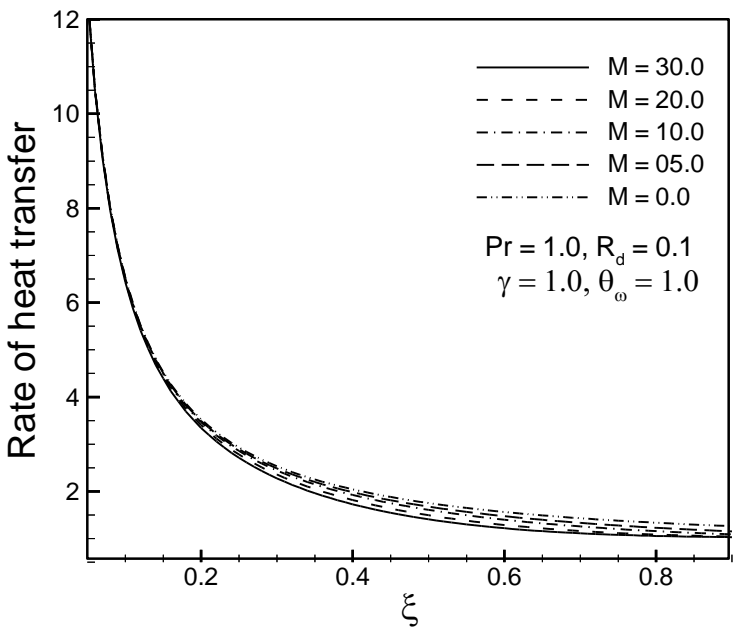

(b)

Fig 7.(a) Skin friction and (b) rate of heat transfer for different values of heat generation parameter $Q$ with others fixed parameters.

Numerical values of rate of heat transfer $N u_{x}$ and skin friction coefficient $C_{f x}$ are calculated from Equations (13) from the surface of the vertical porous plate. Numerical values of $C_{f x}$ and $N u_{x}$ are shown in Table 1.

In the below table the values of skin friction coefficient $\mathrm{C}_{f x}$ and Nusselt number $N u_{x}$ are recorded to be 0.25075 , $0.29810,0.39677$ and 0.99889 and $0.99564,1.00510,1.00388$ and 1.03770 for $\gamma=0.5$ respectively which occur at the same point $\xi=1.7$. Here, it observed that at $\xi=1.7$, the skin friction decreases by $74.89 \%$ and Nusselt number $N u_{\mathrm{x}}$ decreases by $4.13 \%$ as the MHD parameter $M$ changes from 0.0 to 30.0.

Table:1 Skin friction coefficient and rate of heat transfer against $x$ for different values of heat generation parameter $Q$ with other controlling parameters $\operatorname{Pr}=1.0, R_{d}=0.1, \theta_{\mathrm{w}}=1.1$.

\begin{tabular}{ccccccccc}
\hline \multirow{2}{*}{$\xi$} & \multicolumn{2}{c}{$M=30.0$} & \multicolumn{2}{c}{$M=20.0$} & \multicolumn{2}{c}{$M=5.0$} & \multicolumn{2}{c}{$M=0.0$} \\
\cline { 2 - 8 } & $C_{f x}$ & $N_{u x}$ & $C_{f x}$ & $N_{u x}$ & $C_{f x}$ & $N_{u x}$ & $C_{f x}$ & $N_{u x}$ \\
\hline 0.01 & 0.00698 & 61.05675 & 0.00698 & 61.06249 & 0.00698 & 61.07110 & 0.00698 & 61.07397 \\
0.05 & 0.03479 & 12.53074 & 0.03486 & 12.54781 & 0.03496 & 12.57349 & 0.03499 & 12.58207 \\
0.10 & 0.06850 & 6.43424 & 0.06899 & 6.46552 & 0.06973 & 6.51282 & 0.06998 & 6.52869 \\
0.50 & 0.07527 & 1.40758 & 0.25805 & 1.49423 & 0.32120 & 1.67762 & 0.35005 & 1.75275 \\
1.00 & 0.22991 & 1.01766 & 0.29955 & 1.02255 & 0.48923 & 1.09862 & 0.67436 & 1.20826 \\
1.50 & 0.25147 & 1.00576 & 0.29856 & 1.00488 & 0.51757 & 1.00624 & 0.92699 & 1.06340 \\
1.70 & 0.25053 & 0.99564 & 0.29810 & 1.00510 & 0.51655 & 1.00384 & 0.99889 & 1.03770 \\
\hline
\end{tabular}

\section{Comparison of the results}

In order to verify the accuracy of the present work, the values of Nusselt number and skin friction for $Q=0$, $R_{d}=0.05 . \operatorname{Pr}=1.0, \gamma=0$ and various surface temperature $\theta_{w}=1.1, \theta_{w}=2.5$ at different position of $\xi$ are compared with Hossain [9] as presented in Table 2. The results are found to be in excellent agreement.

Table 2 : Comparison of present numerical results of $C_{f x}$ and $N u_{x}$ for the values of prandtl number $\operatorname{Pr}=1.0$, radiation parameter $R_{d}=0.05$, for surface temperature $\theta w=1.1$ and $\theta w=1.5$ without the effect of magnetohydridynamic and heat generation parameter with Hossain et al. [9].

\begin{tabular}{|c|c|c|c|c|c|c|c|c|}
\hline \multirow{3}{*}{$\xi$} & \multicolumn{4}{|c|}{$\theta \mathrm{w}=1.1$} & \multicolumn{4}{|c|}{$\theta w=2.5$} \\
\hline & \multicolumn{2}{|c|}{ Hossain } & \multicolumn{2}{|c|}{ Present } & \multicolumn{2}{|c|}{ Hossain } & \multicolumn{2}{|c|}{ Present } \\
\hline & $C_{f x}$ & $N_{u x}$ & $C_{f x}$ & $N_{u x}$ & $C_{f x}$ & $N_{u x}$ & $C_{f x}$ & $N_{u x}$ \\
\hline 0.1 & 0.0655 & 6.4627 & 0.06535 & 6.48306 & 0.0709 & 0.0709 & 0.0709 & 0.0709 \\
\hline 0.2 & 0.1316 & 3.4928 & 0.13138 & 3.50282 & 0.1433 & 0.1433 & 0.1433 & 0.1433 \\
\hline 0.4 & 0.2647 & 2.0229 & 0.26408 & 2.03018 & 0.2917 & 0.2917 & 0.2917 & 0.2917 \\
\hline 0.6 & 0.3963 & 1.5439 & 0.39519 & 1.55522 & 0.4423 & 0.4423 & 0.4423 & 0.4423 \\
\hline 0.8 & 0.5235 & 1.3247 & 0.52166 & 1.32959 & 0.5922 & 0.5922 & 0.5922 & 0.5922 \\
\hline 1.0 & 0.6429 & 1.1995 & 0.64024 & 1.20347 & 0.7379 & 0.7379 & 0.7379 & 0.7379 \\
\hline 1.5 & 0.8874 & 1.0574 & 0.88192 & 1.06109 & 1.0613 & 1.0613 & 1.0613 & 1.0613 \\
\hline
\end{tabular}




\section{Conclusion}

For different values of relevant physical parameters including the viscosity parameter $\gamma$, the effect of radiation on MHD natural convection flow from a porous vertical plate has been investigated. The governing boundary layer equations of motion are transformed into a non-dimensional form and the resulting non-linear systems of partial differential equations are reduced to local non-similarity boundary layer equations, which are solved numerically by using implicit finite difference method together with the Keller-box scheme. From the present investigation the following conclusions may be drawn:

- $\quad$ Significant effects of MHD parameter $M$ and viscosity parameter $\gamma$ on velocity and temperature profiles as well as on skin friction coefficient $C_{f x}$ and the rate of heat transfer $N u_{x}$ have been found in this investigation but the effect of MHD parameter $M$ and viscosity parameter $\gamma$ on rate of heat transfer is more significant. An increase in the values of viscosity parameter $\gamma$ leads to the velocity decrease and the temperature profiles decrease, the local skin friction coefficient $C_{f x}$ increase and the local rate of heat transfer $N u_{x}$ decreases at different position of $\xi$ for $P r$ $=1.0$.

- For increasing values of Prandtl number $\operatorname{Pr}$ leads to decrease the velocity profile, the temperature profile and the local skin friction coefficient $C_{f x}$ but the local rate of heat transfer $N u_{x}$ increases.

- An increase in the values of $M$ leads to increase the temperature profiles and the velocity profiles, the local skin friction coefficient $C_{f x}$ and the local rate of heat transfer $N u_{x}$ decreases.

\section{Nomenclatures}

$a_{r} \quad$ Rosseland mean absorption co-efficient

$C_{f} \quad$ Local skin friction coefficient

$C_{p} \quad$ Specific heat at constant pressure

$f \quad$ Dimensionless stream function

$g \quad$ Acceleration due to gravity

$k \quad$ Thermal conductivity

$N u_{x} \quad$ Local Nusselt number

$\mathrm{Pr} \quad$ Prandtl number

$Q \quad$ Heat generation parameter

$q_{w} \quad$ Heat flux at the surface

$q_{c} \quad$ Conduction heat flux

$q_{r} \quad$ Radiation heat flux

$R_{d} \quad$ Radiation parameter

$T \quad$ Temperature of the fluid in the boundary layer

$T_{\infty} \quad$ Temperature of the ambient fluid

$T_{w} \quad$ Temperature at the surface

$(u, v) \quad$ Dimensionless velocity components along $(x, y)$ axes

$(x, y) \quad$ Axis in the direction along and normal to the surface respectively

$V \quad$ Wall suction velocity

\section{Greek symbols}

$\begin{array}{cl}\alpha & \text { Equal to } 4 / 3 R_{d} \\ \beta & \text { Coefficient of thermal expansion } \\ \Delta & \text { Equal to } \theta_{w}-1 \\ \Delta T & \text { Equal to } T_{w}-T_{\infty} \\ \eta & \text { Similarity variable } \\ \theta & \text { Dimensionless temperature function } \\ \theta_{w} & \text { Surface temperature parameter } \\ \mu & \text { Viscosity of the fluid } \\ \nu & \text { Kinematic viscosity } \\ \xi & \text { Similarity variable } \\ \rho & \text { Density of the fluid } \\ \sigma & \text { Stephman-Boltzman constant } \\ \sigma & \text { Scattering co-efficient } \\ \mu_{f} & \text { Absolute Viscosity at the film temperature } \\ \tau & \text { Coefficient of skin friction } \\ \tau_{\mathrm{w}} & \text { Shearing stress } \\ \psi & \text { Non-dimensional stream function }\end{array}$

\section{Subscripts}

$w \quad$ wall conditions

$\infty \quad$ Ambient temperature

\section{References}

[1] J. H. Merkin, Free convection with blowing and suction, International journal of heat and mass transfer, Vol.15, No.1, 1972, pp. 989-999.

[2] H. T. Lin, W. S. Yu, Free convection on horizontal plate with blowing and suction. Transactions on ASME journal of Heat Transfer, Vol.110, No.3, 1988, pp. 793-796.

[3] M. A. Hossain, K. Khanafer, K. Khafai. The effect of radiation on free convection flow with variable viscosity from a porous vertical plate, International Journal of Thermal science, Vol.40, No.2, 1999, pp. 115-124.

[4] M. A. Hossain, M. S. Munir, D. A. S. Rees, Flow of viscous incompressible fluid with temperature dependent viscosity and thermal conductivity past a permeable wedge with variable heat flux, Int. Journal of Thermal science, Vol.39, No.6, 2000, pp. 635-644.

[5] M. A. Hosain, H.S. Takhar, Radiation effect on mixed convection along a vertical plate with uniform surface Temparatue, Journal of Heat and Mass Transfer, Vol.31, No.4, 2001, pp. 243-248. 
[6] M. M. Molla, M. A. Hossain, L.S. Yao, Natural convection flow along a vertical wavy surface with uniform surface temperature in presence of heat generation/ absorption, International Journal of Thermal Science, Vol.43, No.2, 2004, pp. $157-163$.

[7] Tahmina Akhter, Effect of Radiation on Natural Convection Flow on a Sphere with Isothermal surface and uniform Heat Flux, M.Phil Thesis, Department of Mathematics, Bangladesh University of Engineering and Technology (BUET), Dhaka, Bangladesh, 2007.

[8] Md. Miraj Ali, Numerical Study of Radiation on Natural Convection Flow on a Sphere with Heat Generation, M.Phil Thesis, Department of Mathematics, Bangladesh University of Engineering and Technology (BUET), Dhaka, Bangladesh, 2007.

[9] M. A. Hossain, M. A. Alim, D. A. S. Rees, The effect of radiation on free convection flow from a porous vertical plate, International Journal of Heat and Mass Transfer, Vol.42, No.1, 1999, pp. 181-191.

[10] K. Vejravelu, A. Hadjinicolaou, Heat transfer in a viscous fluid over a stretching sheet with viscous dissipation and internal heat generation, International Communications in Heat and Mass Transfer, Vol.20, No.3, 1993, pp. 417-430.

[11] M. M. Molla, M. A. Hossain, M. A. Taher, Magnetohydrodynamic natural convection flow on a sphere with uniform heat flux in presence of heat generation, Acta Mechanica, Vol.186, 2006, pp. 75-86.

[12] J. Gary, D.R. Kassory, H. Tadjeran, A. Zebib, The effect of significant viscosity variation on convective heat transport in water-saturated porous media, Journal of Fluid Mechanics, Vol.117, 1982, pp. 233-249.

[13] K.N. Mehta, S. Sood, Transient free convection flow with temperature dependent viscosity in a fluid saturated porous medium, International Journal of Engineering and Science, Vol.30, 1992, pp. 1083-1087.

[14] N.G. Kafoussius, E.W. Williams, The effect of temperature-dependent viscosity on the free convective laminar boundary layer flow past a vertical isothermal flat plate, Acta Mechanica, Vol.110, 1995, pp. 123-137.

[15] N.G. Kafoussius, D.A.S. Rees, Numerical study of the combined free and forced convective laminar boundary layer flow past a vertical isothermal flat plate with temperature dependent viscosity, Acta Mechanica, Vol.127, 1998, pp. 39-50.

[16] M.A. Hossain, S. Kabir, D.A.S. Rees, Natural convection of fluid with variable viscosity from a heated vertical wavy surface, Journal of Applied Mathematics and Physics(ZAMP), Vol. 53, No.1, 2002, pp. 48-52.

[17] M.A. Hossain, M.S. Munir, Mixed convection flow from a vertical flat plate with temperature dependent viscosity, International Journal of Thermal Science, Vol.39, No.2, 2000, pp. 173-183.

[18] V.P. Carey, J.C. Mollendorf, Natural convection in liquid with temperature dependent viscosity, Proceedings of $6^{\text {th }}$ International Heat Transfer Conference, Toronto, Vol.2, 1978, pp. 211-217.

[19] M. Necati Özisik, Radiative Transfer and Interactions with Conduction and Convection, New York : John Wiley, 1973.

[20] H.B. Keller, Numerical methods in boundary layer theory, Annual Review of Fluid Mechanics, Vol.10, 1978 , pp. 417-433.

[21] T. Cebeci, P. Bradshaw, Physical and Computational Aspects of Convective Heat Transfer, Springer, New York, 1984. 